\title{
The Noise Generated by a Landing Gear Wheel with Hub and Rim Cavities
}

\author{
Meng Wang ${ }^{\mathrm{a}}$, David Angland ${ }^{\mathrm{a}, *}$, Xin Zhang ${ }^{\mathrm{b}}$ \\ ${ }^{a}$ Faculty of Engineering and the Environment \\ University of Southampton, Southampton, SO16 7QF, UK \\ ${ }^{b}$ Department of Mechanical and Aerospace Engineering \\ The Hong Kong University of Science and Technology, Hong Kong SAR
}

\begin{abstract}
Wheels are one of the major noise sources of landing gears. Accurate numerical predictions of wheel noise can provide an insight into the physical mechanism of landing gear noise generation and can aid in the design of noise control devices. The major noise sources of a $33 \%$ scaled isolated landing gear wheel are investigated by simulating three different wheel configurations using high-order numerical simulations to compute the flow field and the FW-H equation to obtain the far-field acoustic pressures. The baseline configuration is a wheel with a hub cavity and two rim cavities. Two additional simulations are performed; one with the hub cavity covered (NHC) and the other with both the hub cavity and rim cavities covered (NHCRC). These simulations isolate the effects of the hub cavity and rim cavities on the overall wheel noise. The surface flow patterns are visualised by shear stress lines and show that the flow separations and attachments on the side of the wheel, in both the baseline and the configuration with only the hub cavity
\end{abstract}

\footnotetext{
${ }^{*}$ Corresponding author

Email address: d.angland@soton.ac.uk
} 
covered, are significantly reduced by covering both the hub and rim cavities. A frequency-domain $\mathrm{FW}-\mathrm{H}$ equation is used to identify the noise source regions on the surface of the wheel. The tyre is the main low frequency noise source and shows a lift dipole and side force dipole pattern depending on the frequency. The hub cavity is identified as the dominant middle frequency noise source and radiates in a frequency range centered around the first and second depth modes of the cylindrical hub cavity. The rim cavities are the main high-frequency noise sources. With the hub cavity and rim cavities covered, the largest reduction in Overall Sound Pressure Level (OASPL) is achieved in the hub side direction. In the other directivities, there is also a reduction in the radiated sound.

Keywords:

aeroacoustics, high-order CAA, landing gear wheel, cavity noise, depth modes

\section{Introduction}

Landing gears are recognized as one of the most significant contributors to airframe noise for commercial aircraft in the approach configuration [1]. A landing gear is an assembly of a large number of components with different 5 sizes and shapes. Wheels are one of the major large-scale landing gear components, which can be considered as the most significant noise sources for simplified two-wheel nose landing gears [2] and important noise contributors for four-wheel main landing gears [3]. Numerical studies of wheel noise can provide a useful insight into the landing gear noise generation mechanisms and can aid in the design of noise reduction treatments. 
A simplified landing gear wheel can be considered as a short aspect ratio circular cylinder. Zdravkovich et al. [4] performed experiments to investigate the flow features past free-end circular cylinders of spanwise length to diameter ratio $W / D$ between 1 and 10 . They found two counter-rotating streamwise vortices formed by the flow separation at the cylinder ends. The vortices convect downstream as a streamwise vortex pair. The highly three dimensional flow near the ends of the cylinders was found to interrupt the vortex shedding resulting in more broadband noise compared to the tonal noise observed in the case of cylinders with large aspect ratios $(W / D \gg 10)$.

20 Several experimental and numerical tests have been performed to study the flow features and far-field acoustics of landing gear wheels. Lazos $[5,6]$ analysed the mean flow features around the wheels of a simplified four-wheel landing gear. The flow separation and attachment regions on the wheel surface were considered to be potential sources of noise [6]. Neuhart et al. [7] performed aerodynamic experiments of a Gulfstream G550 nose landing gear. They reported that the hub area on the wheels might be one of the stronger noise sources due to the high levels of turbulent kinetic energy and pressure perturbations found around the hub area. Yokokawa et al. [8] measured the far-field acoustics generated from a two-wheel main landing gear. They found that the dominant noise sources were the tyre and the sidebrace, compared to the cylinder, the axle, the torque link and the landing gear door. In a four-wheel Rudimentary Landing Gear (RLG) test, complex flow interactions were found between the upstream and downstream wheels $[9,10]$, which might be a significant noise source. Liu et al. [2] performed 35 high-order simulations of a two-wheel nose landing gear and found that the 
wheel noise dominated the strut noise and the axle noise. They observed that more wheel noise radiated towards the sideline direction.

Cylindrical cavities are present on realistic landing gear wheels. These cylindrical cavities can generate tonal and broadband noise. An experimental aeroacoustic study of a high-fidelity six-wheel landing gear performed by Jaeger et al. [11] showed that a reduction of $3 \mathrm{~dB}$ in the far-field acoustic SPL can be achieved by covering the hub cavity with a flat plate [11]. In the experiments performed as part of the LAnding Gear nOise database for CAA validatiON (LAGOON) project, two tonal peaks were found in the sideline 45 direction for a generic two-wheel landing gear with two facing rim cavities on the wheels, and they are also confirmed by numerical simulations [12]. The tones are generated by the interaction of the shear layer between the wheels with the acoustic resonance of the rim cavities. These two tones were further investigated numerically by Casalino et al. [13], who found that the first tone was related to a plane wave corresponding to the floor-to-floor cavity distance, while the second tone was from an azimuthal mode of the wheel cavities [13]. Zhang et al. [14] performed aerodynamic and aeroacoustic experiments of an isolated high-fidelity landing gear wheel including a tyre, a sidewall, a hub, a hub cavity and rim cavities. They found that the wheel noise is characterized by broadband middle frequency noise centred around $630 \mathrm{~Hz}$ and $1250 \mathrm{~Hz}$, which are fixed by the geometry dimensions and does not scale with flow velocities. Wang et al. [15] conducted numerical simulations with the same geometry used by Zhang et al. [14] and found that the noise at $630 \mathrm{~Hz}$ and $1250 \mathrm{~Hz}$ is generated by the first and second depth modes 60 of the hub cavity and the OASPL of the noise at the hub side is $4 \mathrm{~dB}$ 
higher than the other sideline direction. The opposite facing rim cavities in the numerical simulations by Casalino et al. [13] and the hub cavity in the experiments by Jaeger et al. [11], Zhang et al. [14] and numerical simulation by Wang et al. [15] share shallow cylindrical cavity geometries. It has been shown by Marsden et al. [16] that the dominant mechanism driving the flow around relatively deep cylindrical cavities is the interaction of aerodynamic flow with the cavity resonance of the depth modes, which is different from the acoustic feedback in the Rossiter's mode [17]. However, for shallow cylindrical cavities, which is the case of the hub cavity in this work, they reported that tones are not as distinguishable compared to deep cylindrical cavities, and the far-field acoustics are generally more broadband [18]. Thus, the sound generated by a hub cavity on a landing gear wheel is expected to be broadband rather than tonal.

The baseline geometry used in the simulations is the same as the one used in the experiments by Zhang et al. [14]. The validation of the numerical methodology against experimental data for the baseline configuration was presented by Wang et al. [15]. The validation is not repeated in this paper. In this current work, the effects of the hub cavity and the rim cavities on the wheel noise are isolated by covering them in two different simulations and comparing them to the baseline simulation. The results from the baseline configuration are also analysed to give an insight into the sound generation mechanisms and their radiation characteristics. The wheel geometry and details of the grid generation are provided in Section 2. Section 3 describes the numerical methodology and the computational setup in the simulations. ${ }_{85}$ The aerodynamic and acoustic simulation results are presented in Section 4, 
focussing on the effects of the hub cavity and rim cavities on the near-field flows and far-field acoustics.

\section{Model detail and computational grids}

A $33 \%$ scaled isolated landing gear wheel from the Technology Strategy a short aspect ratio circular cylinder and can be compared with the NHC configuration to investigate the noise generated from the rim cavities.

The numerical simulations were performed on multi-block structured grids. 

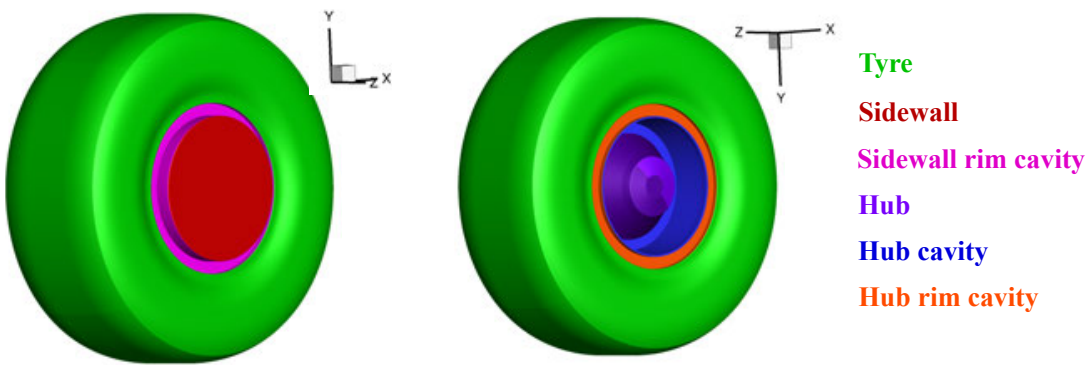

Figure 1: The geometry of the CADWIE wheel in the simulations. The origin of the axes is at the wheel centre.

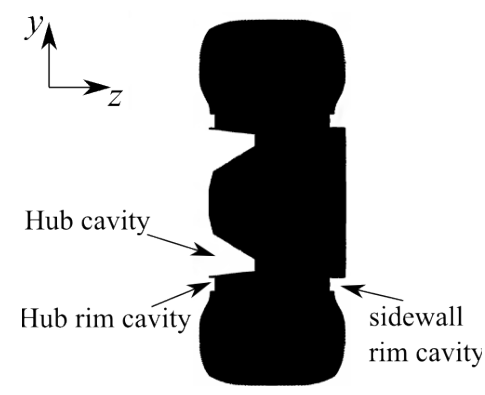

(a) Baseline.

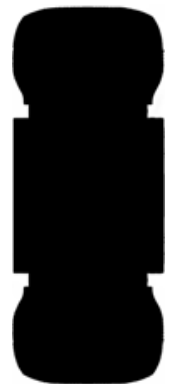

(b) NHC.

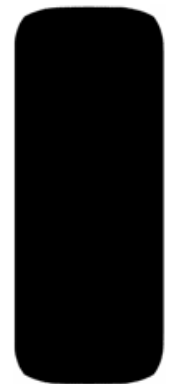

(c) NHCRC.

Figure 2: The cross section of three geometry configurations on the $x / D=0$ plane. (a) Baseline geometry; (b) No Hub Cavity (NHC) geometry; (c) No Hub Cavity and Rim Cavities (NHCRC) geometry. 
The computational domain is shown in Figure 3 with the origin of the axes at the centre of the wheel. The computational domain extends $5 D, 4 D$ and $16 D$ in the upstream, above and below, and downstream directions, respectively. The spanwise length of the domain is $8 D$. In the boundary layer region, the value of the wall distance $y^{+}$is smaller than 2.0 and a grid stretching ratio of 1.15 in the wall normal direction is used to relax the grid distribution. For the baseline geometry, the hub cavity walls are meshed by a cylindrical grid with 300, 150 and 85 grid points in the azimuthal, radial and depth directions. The same mesh resolution is used in the sidewall region. There are 60 grid points distributed along the width of the wheel. The total number of grid points is $25 \times 10^{6}$. A grid sensitivity was performed by Wang et al. [15], and showed that this grid resolution was adequate to provide good agreement to the aerodynamic and acoustic experimental results. For the NHC and NHCRC configurations, the mesh resolutions on the surface of the tyre and sidewall are the same as the baseline case. Figure 4 shows the mesh topology and grid distributions around the CADWIE wheel for the baseline configuration. The wheel is surrounded by an O-type mesh in the near field, which transitions to a H-type mesh away from the wheel. Details of the mesh around the sidewall rim cavity and the hub cavity are shown in Figures 4(c) and $4(\mathrm{~d})$.

\section{Numerical methods and computational setup}

The simulations were performed using an in-house high-order finite-difference solver SotonCAA. The three-dimensional Navier-Stokes equations are solved with the Delayed Detached Eddy Simulation (DDES) turbulence model [19] 


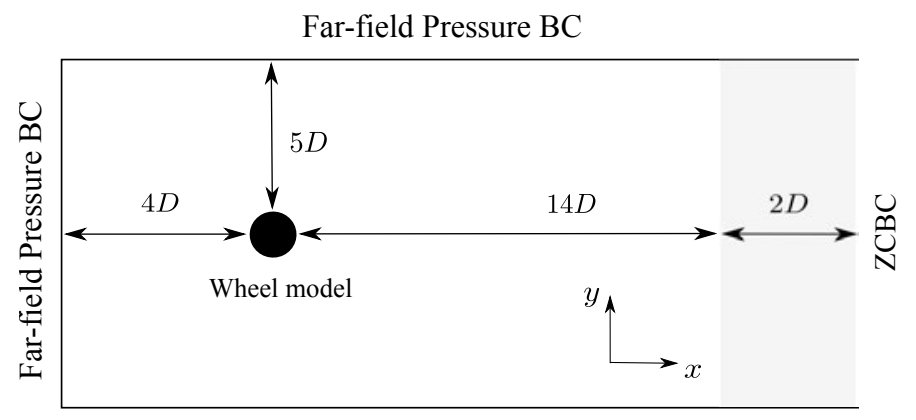

Far-field Pressure BC

Figure 3: The computational domain and boundary conditions in the simulations.

in generalized coordinates using high-order optimized penta-diagonal finitedifference schemes [20]. Compact filters [21] are used for numerical stability. At block interfaces with grid metric discontinuities, a finite volume method is applied to reduce grid-induced errors [22]. The inflow boundary condition is a far-field pressure condition based on Riemann invariants. The non-reflecting outflow boundary condition is a Zonal Characteristic Boundary Condition (ZCBC) [23], which can significantly reduce pressure perturbations generated by vortical waves passing through the outlet [23]. The eddy viscosity ratio at the inlet is 5 , resulting in a fully turbulent boundary layer. The experimental database used to validate the current simulations is obtained from free transition, and it is demonstrated that at this scale, the experimental measurements are relatively insensitive to the transition treatment [14]. Noslip isothermal wall boundary conditions are imposed on the wheel surface. A second-order implicit time-stepping method with Newton-like subiterations is used to march the solution in time [24]. The far-field acoustics are computed using the Ffowcs-Williams and Hawkings (FW-H) method, based on the Farassat 1A formulation [25]. The FW-H integral surface in the simu- 


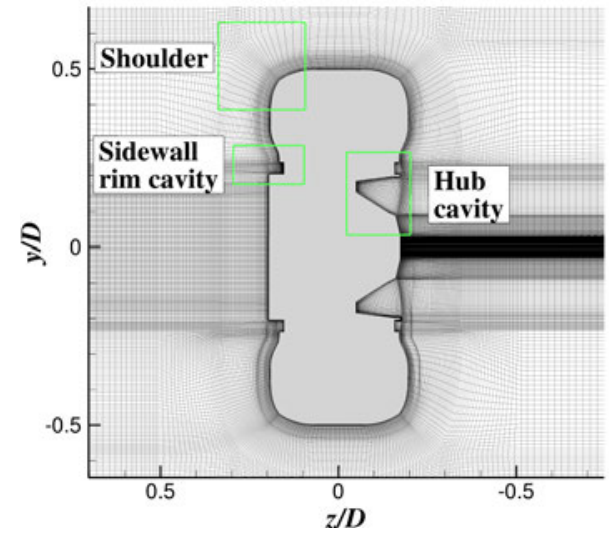

(a) Mesh around the CADWIE wheel on $x / D=0$ plane.

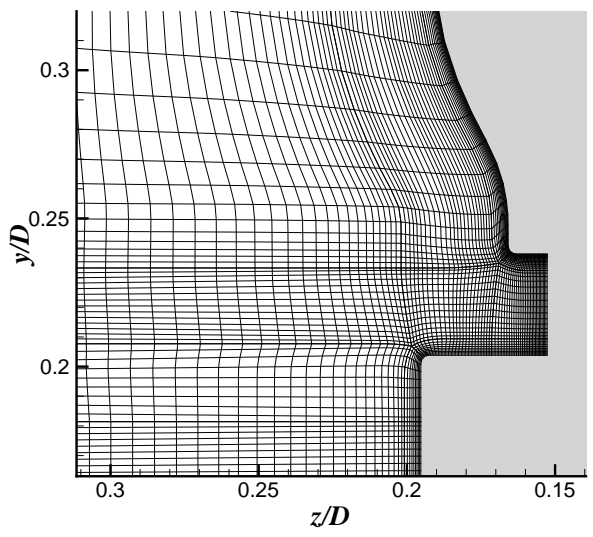

(c) Mesh around the sidewall rim cavity.

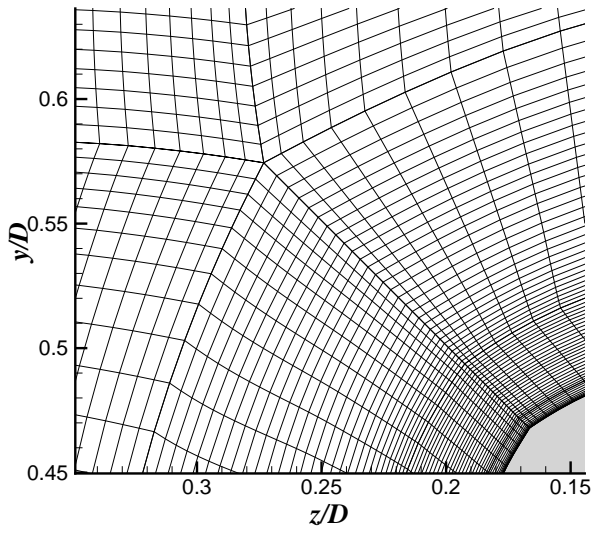

(b) Mesh around the shoulder.

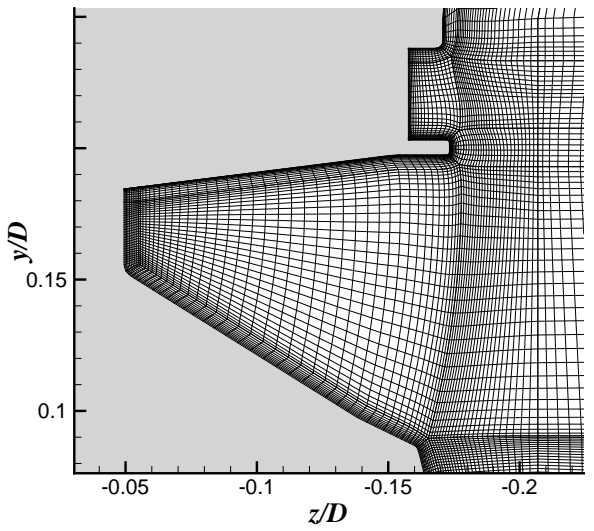

(d) Mesh around the hub cavity.

Figure 4: Mesh topology and grid distribution around the CADWIE wheel. 
lations is coincident with the solid surface of the wheel. The solid FW-H integral surface is able to reduce the risk of noise contamination by large vortical wave fluctuations convecting through the surface [26].

The freestream quantities are $\rho_{\infty}=1.22 \mathrm{~kg} / \mathrm{m}^{3}, p_{\infty}=99456 \mathrm{~Pa}$ and

155 are isolated by covering them in two different simulations and comparing them to the baseline simulation. 


\subsection{Instantaneous flow field}

175

The instantaneous flow features on the side of the wheel for the three different configurations are shown in Figure 5. For the baseline configuration in Figure 5(a), the flow first separates from the tyre at point $\mathrm{F}$ and forms a vortex in the hub rim cavity. After separation, Kelvin-Helmholtz (K-H) instabilities in the shear layer begin to develop and the shear layer interacts with the flow recirculation region inside the hub cavity. The highly turbulent flow in the hub cavity region is visualised by the plot of $Q$-criterion shown in Figure 5(a). When the hub cavity is covered in the NHC configuration, the separated flow, which separates at point F, reattaches to the sidewall, as shown in Figure 5(b). This reduces the turbulent flow generated by the hub cavity. In the NHCRC configuration, due to the absence of the hub cavity and rim cavities, the flow is fully attached to the sidewall (Figure $5(\mathrm{c}))$. The turbulent flow associated with the hub cavity and rim cavities is eliminated. The effect of these flowfield changes on the far-field acoustics will be quantified in Section 4.4.

\subsection{Mean surface shear stress lines}

Flow separation and attachment can be considered as potential noise sources [6]. The surface shear stress lines are used to characterise the flow separation and attachment. Following the work of Lazos [6], the basic patterns of separation/attachment in the shear stress lines of landing gear wheels can be characterised into stable/unstable regular node, negative/positive open bipartition, saddle of separation/attachment, which are summarised by La$\operatorname{zos}[6]$. 


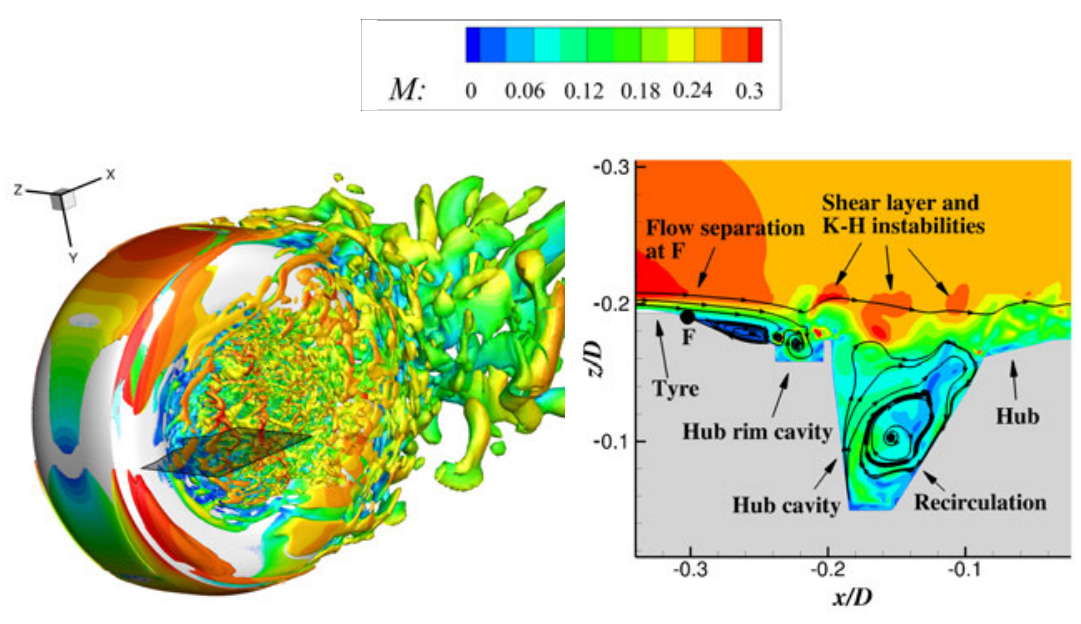

(a) Baseline.
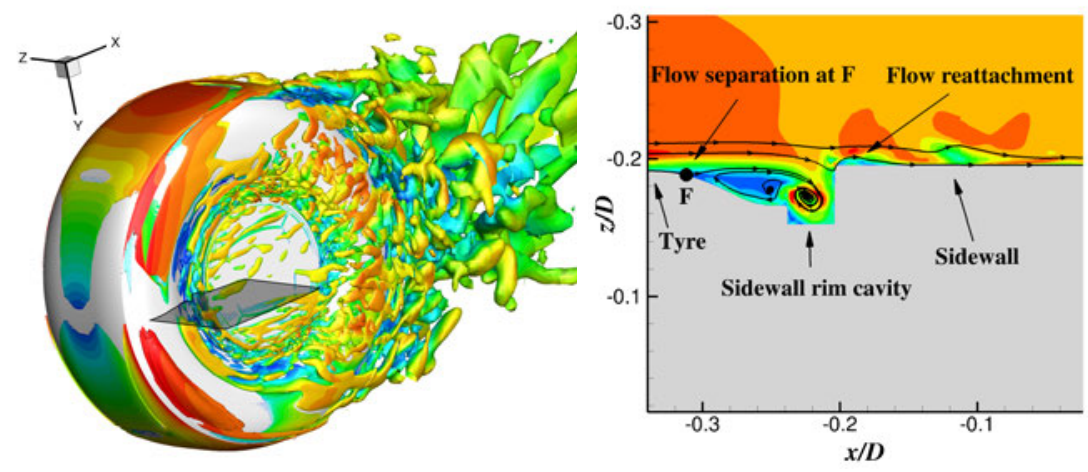

(b) NHC.
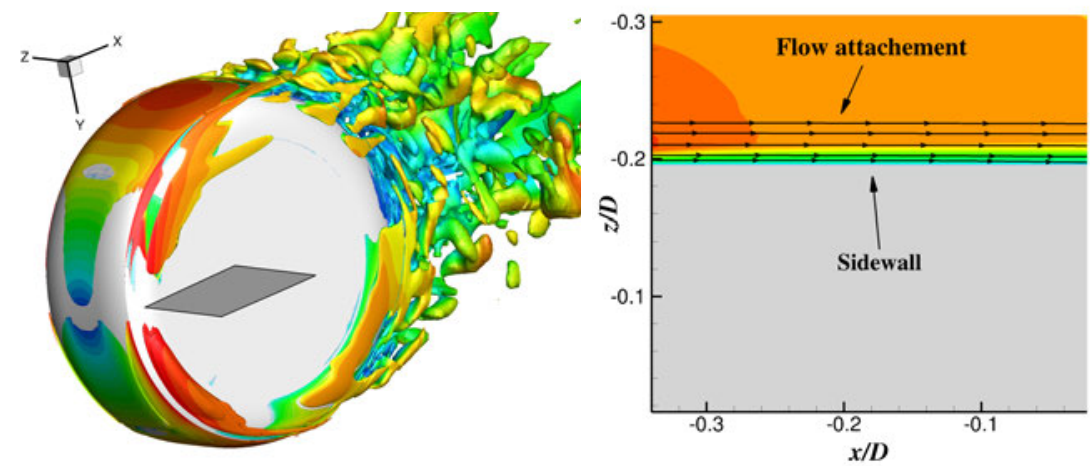

(c) NHCRC.

Figure 5: Left: instantaneous $Q$-criterion, where $Q=10 \times U_{\infty}^{2} / D^{2}$ and is coloured by the local Mach number. Right: instantaneous local Mach number and flow features on the $y / D=0$ plane highlighted on the left. 
Figure 6 shows the surface flow features on the side of the wheel with the main flow features highlighted. At the leading edge of the baseline configuration in Figure 6(b), a favourable pressure gradient exists from the stagnation point A to the end of the surface curvature B. This is evidenced by the surface pressure contours around the shoulder of the wheel. Separation occurs at the saddle of separation B. Just downstream of this point, the flow reattaches at the saddle D. Saddle B and D form negative and positive closed bipartitions, respectively. Between these two points there is a recirculation region where the surface flow direction is predominantly toward the leading edge of the wheel. Further downstream of D, the flow moves towards the hub cavity and separates at $\mathrm{F}$. The separation point $\mathrm{F}$ is also highlighted in the plot of instantaneous local Mach number in Figure 5(a). The negative open bipartition extends from $\mathrm{F}$ along the leading edge of the hub rim cavity to the downstream edge of the wheel G. F-G forms a separation line, which can also be observed in the experimental surface oil flow image in Figure 6(a). Downstream of this separation line, the separated flow is highly turbulent as shown in Figure 5(a). Further downstream of the saddle of separation $\mathrm{F}$, the flow reattaches at the saddle of attachment $\mathrm{H}$. On the downstream edge of the hub rim cavity, the two positive open bipartite lines converge at the saddle of attachment I. The flow features of the NHC configuration are similar to the baseline case, except that the separated flow from the upstream rim cavity reattaches to the sidewall at $\mathrm{J}$ and then remains attached until the downstream rim cavity. For the NHCRC configuration, the flow is fully attached to the sidewall after the saddle of attachment D. The complexity of flow separation and attachment patterns in the baseline and $\mathrm{NHC}$ 
configurations is significantly reduced in the NHCRC configuration.

\subsection{Acoustic results from the baseline geometry}

\section{3 details the analysis of the baseline geometry. The acoustic results of the} other two configurations are presented in Section 4.4 .

\subsubsection{Far-field acoustics}

It has been found in the validation simulations that the depth modes of the hub cavity are present in the far-field acoustics [15]. The depth modes are given by,

$$
f_{i}=\frac{i c}{4 h^{\prime}}
$$

where $c$ is the speed of sound, $i$ is an integer. The effective depth $h^{\prime}$ taking account of the cavity opening is given by [27],

$$
h^{\prime}=H_{\text {cavity }}+0.8216 \frac{D_{\text {cavity }}}{2},
$$

where $H_{\text {cavity }}$ and $D_{\text {cavity }}$ are the depth and diameter of the hub cavity. The

first and second depth modes of the hub cavity in this geometry are $646 \mathrm{~Hz}$ and $1292 \mathrm{~Hz}$.

The Power Spectral Density (PSD) of the far-field acoustic pressures at the hub side $\left(\psi=90^{\circ}, \phi=180^{\circ}\right)$ and sidewall side $\left(\psi=90^{\circ}, \phi=0^{\circ}\right)$ is 


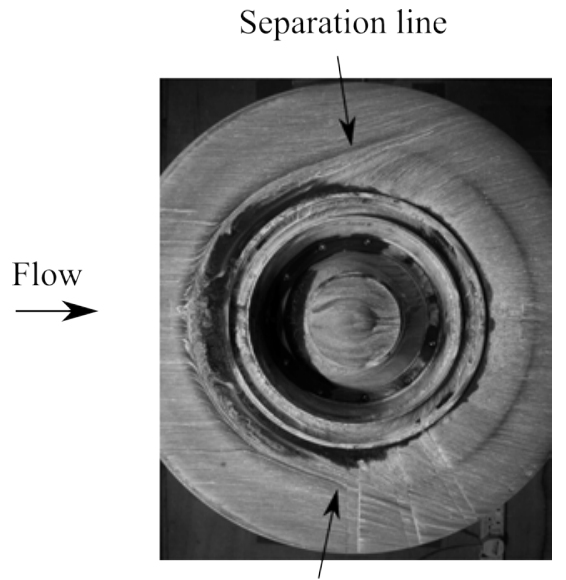

Separation line

(a) Experiment [14].

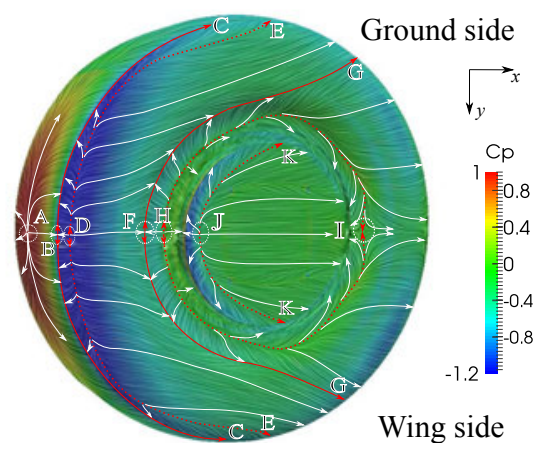

(c) NHC.

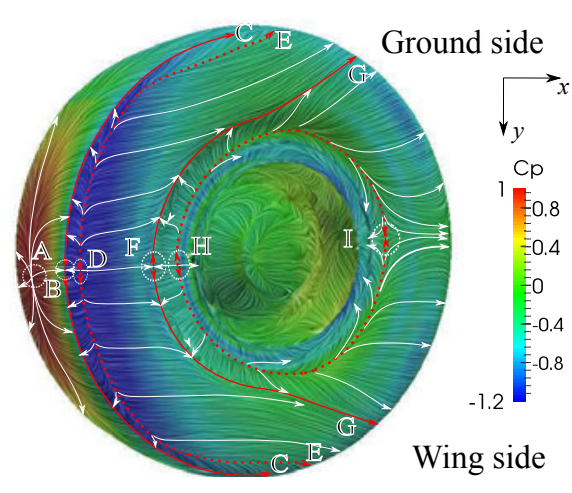

(b) Baseline.

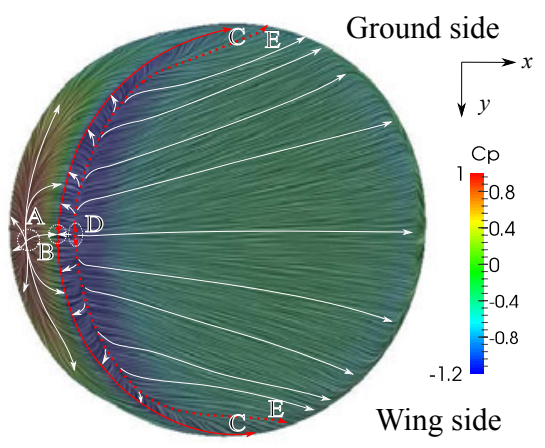

(d) NHCRC.

Figure 6: The surface shear stress lines with typical flow features highlighted. A: unstable regular node; B, F: saddle of separation; D, H, I, J: saddle of attachment; —: positive open bipartite line; - - - : negative open bipartite line. 


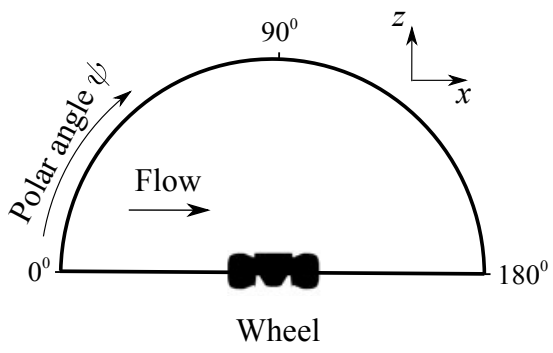

(a) Polar angle $\psi$.

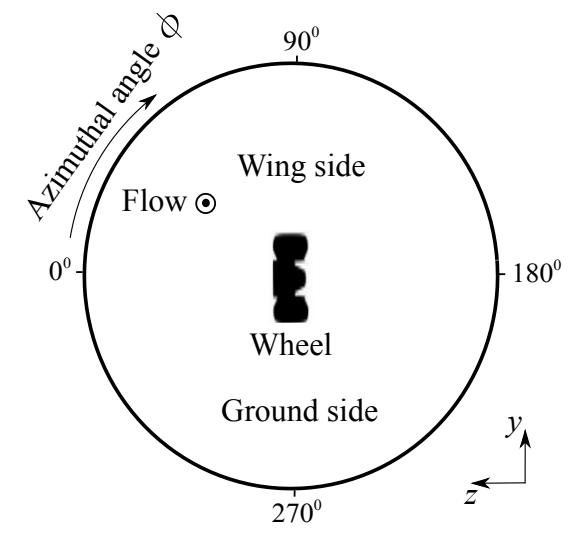

(b) Azimuthal angle $\phi$.

Figure 7: Demonstrations of observer angles, where the hub side observer, sidewall side observer and ground side observer refer to the positions of $\left(\psi=90^{\circ}, \phi=180^{\circ}\right)$, $\left(\psi=90^{\circ}, \phi=0^{\circ}\right)$ and $\left(\psi=90^{\circ}, \phi=270^{\circ}\right)$, respectively.

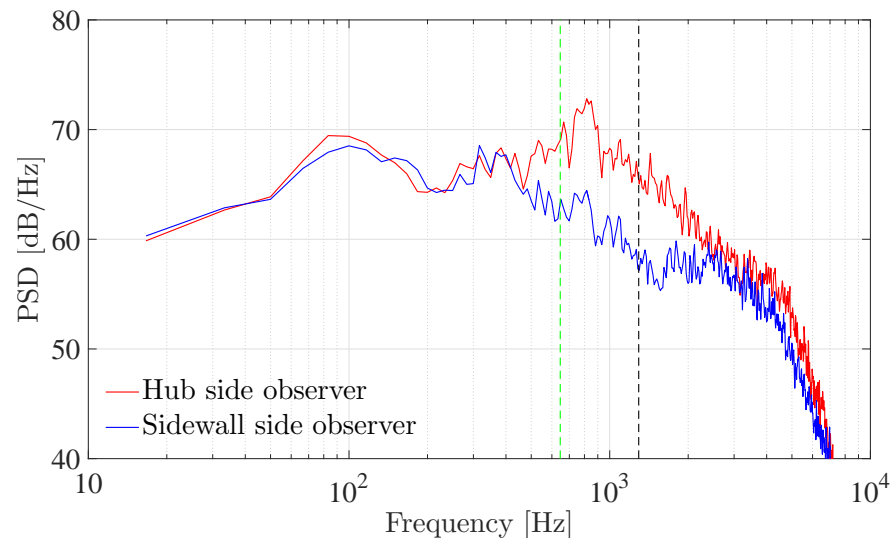

Figure 8: Far-field acoustic spectra at the hub side $\left(\psi=90^{\circ}, \phi=180^{\circ}\right)$ and sidewall side $\left(\psi=90^{\circ}, \phi=0^{\circ}\right)$ of the baseline configuration. - - - first depth mode $(646 \mathrm{~Hz}),-$ - second depth mode $(1292 \mathrm{~Hz})$. 
plotted in Figure 8. The frequency scale is divided into low frequency range $(f<400 \mathrm{~Hz})$, middle frequency range $(400 \mathrm{~Hz}<f<2000 \mathrm{~Hz})$ and high frequency range $(f>2000 \mathrm{~Hz})$. The spectrum of the sidewall side observer is similar to the hub side observer in the low and high frequency ranges, but they are significantly different in the middle frequency range. Compared to the sidewall side, the additional middle frequency noise that radiates towards the hub side observer is due to the presence of the hub cavity. The first and second depth modes of the cylindrical hub cavity are in this middle frequency range. Further evidence that the noise generated in this frequency range is due to the depth modes of the hub cavity resonance is shown in Figure 9. In Figure 9, the experimental far-field acoustic pressure spectra at the hub side observer at three different Mach numbers are shown [14]. The noise sources distributed on the wheel surface can be considered as dipoles, which follow the $M^{6}$ power law [28]. The magnitude of the power has been scaled by this Mach number exponent. The facility noise dominates the low frequency noise in the experiments $(<300 \mathrm{~Hz})$ and is therefore excluded in Figure 9. of the wheel $D$ and the width of the wheel $W$, in Figure 9(a) and against frequency in Figure 9(b). Compared to the Strouhal number scale, a better collapse using the frequency scale is demonstrated in Figure 9(b), particularly in the middle frequency range, indicating that the middle frequency noise is fixed by the dimensions of the geometry. This middle frequency range also corresponds to the first and second depth modes, which provides further evidence that the middle frequency noise is from the depth modes of the hub cavity resonance. 


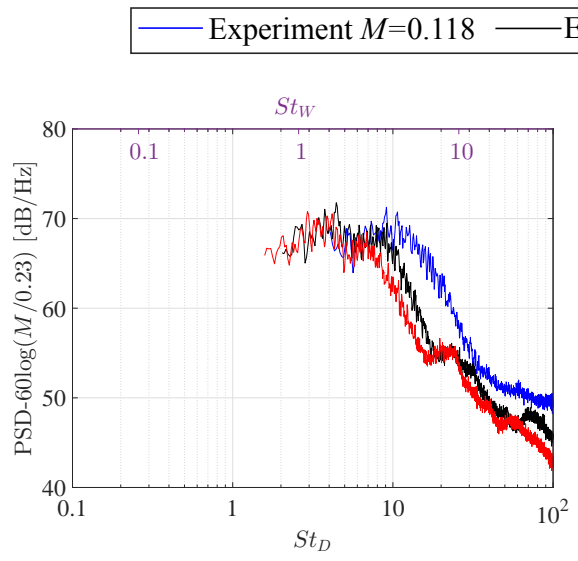

(a) Strouhal number scale.

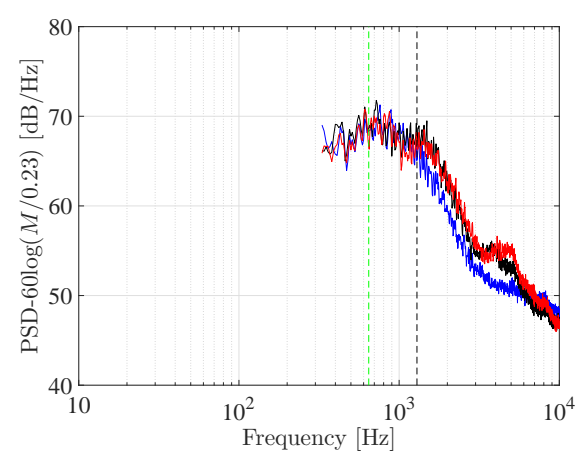

(b) Frequency scale.

Figure 9: The far-field experimental acoustic spectra at the hub side $\left(\psi=90^{\circ}, \phi=180^{\circ}\right)$ in Strouhal number scale and frequency scale. The spectra are collapsed using the $M^{6}$ power law. - - - first depth mode $(646 \mathrm{~Hz})$, - - - second depth mode $(1292 \mathrm{~Hz})$.

\subsubsection{Coherence analysis at the hub cavity depth modes}

The coherence function between wall pressure fluctuations and far-field acoustic pressures is given by,

$$
\gamma^{2}=\frac{S_{p_{w} p_{a b}^{\text {hub }}}^{\text {hat }}}{S_{p_{a}^{\text {hub }} p_{a}^{\text {hub }}} S_{p_{w} p_{w}}}
$$

265 cavity noise is more broadband than the deep cylindrical cavity noise in the 


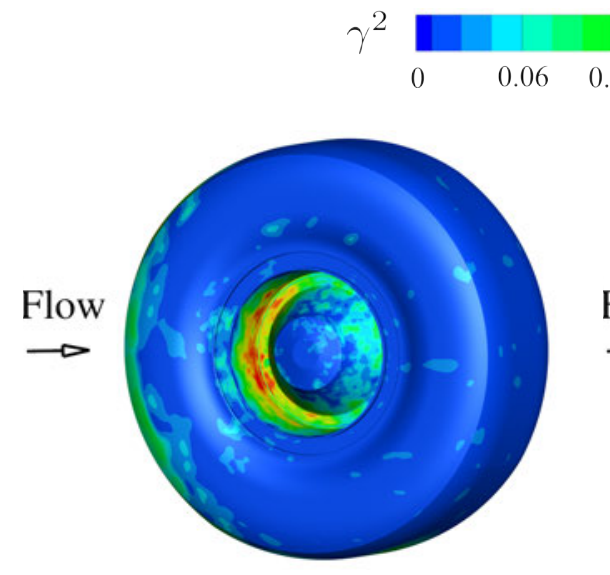

(a) $f=646 \mathrm{~Hz}$.

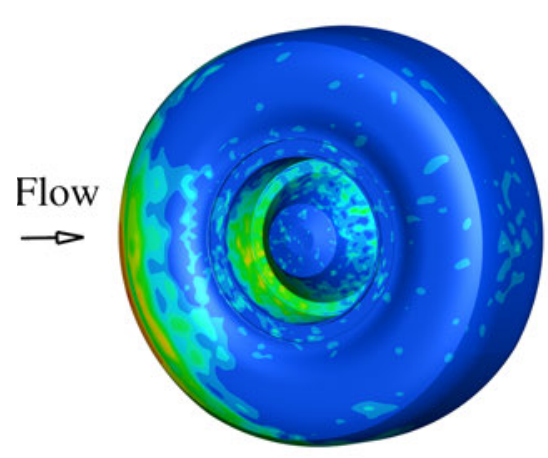

(b) $f=1292 \mathrm{~Hz}$.

Figure 10: The value of coherence function $\gamma^{2}$ between the wall pressure fluctuations and the far-field acoustic pressures at the hub side $\left(\psi=90^{\circ}, \phi=180^{\circ}\right)$.

work by Marsden et al. [16], where a stronger coherence function is present at the depth mode. This broadband cavity noise is consistent with previous work on shallow cylindrical cavities [18].

\subsubsection{Contributions from different parts of the wheel}

For a closed FW-H integral surface containing multiple components, the far-field acoustics can be obtained firstly by using the information on each component individually, which will give an auto power spectral density (PSD), and then by considering the cross correlation between any two components, which will give a cross power spectral density (CPSD). The CPSD is a measure of the coherence and phase shift of the two signals, which can be used to indicate flow and acoustic interactions, as the interactions will give non-zero cross power at the interaction frequency. 
wheel was divided into three components, i.e. the hub cavity (including the surrounding hub rim cavity), the sidewall (including the surrounding sidewall rim cavity) and the tyre. The far-field acoustic pressures in the time domain are computed by,

$$
p(t)=p_{\mathrm{hc}}(t)+p_{\mathrm{s}}(t)+p_{\mathrm{t}}(t)
$$

The far-field pressure spectral power can be obtained by multiplying the complex conjugate to both sides of Equation 5,

$$
\begin{aligned}
S_{p p}= & S_{p_{\mathrm{hc}} p_{\mathrm{hc}}}+S_{p_{\mathrm{s}} p_{\mathrm{s}}}+S_{p_{\mathrm{t}} p_{\mathrm{t}}}+ \\
& S_{p_{\mathrm{hc}} p_{\mathrm{s}}}+S_{p_{\mathrm{s}} p_{\mathrm{hc}}}+S_{p_{\mathrm{hc}} p_{\mathrm{t}}}+S_{p_{\mathrm{t}} p_{\mathrm{hc}}}+S_{p_{\mathrm{s}} p_{\mathrm{t}}}+S_{p_{\mathrm{t}} p_{\mathrm{s}}},
\end{aligned}
$$

where $S_{p_{i} p_{i}}$ is the auto PSD and $S_{p_{i} p_{j}}$ is the CPSD. Since $S_{p_{i} p_{j}}$ is the complex conjugate of $S_{p_{j} p_{i}}$, Equation 6 can be simplified as,

$$
\begin{aligned}
S_{p p}= & S_{p_{\mathrm{hc}} p_{\mathrm{hc}}}+S_{p_{\mathrm{s}} p_{\mathrm{s}}}+S_{p_{\mathrm{t}} p_{\mathrm{t}}}+ \\
& \operatorname{real}\left(2 S_{p_{\mathrm{hc}} p_{\mathrm{s}}}\right)+\operatorname{real}\left(2 S_{p_{\mathrm{hc}} p_{\mathrm{t}}}\right)+\operatorname{real}\left(2 S_{p_{\mathrm{s}} p_{\mathrm{t}}}\right) .
\end{aligned}
$$

The real part of the CPSD can be negative. For visualisation purpose, only the absolute value of the CPSD's real part is plotted in Figure 11.

300 The PSD and CPSD profiles at three observer locations are plotted in Figure 11 against frequency and two Strouhal numbers $\left(S t_{D}\right.$ and $\left.S t_{W}\right)$. Firstly, 


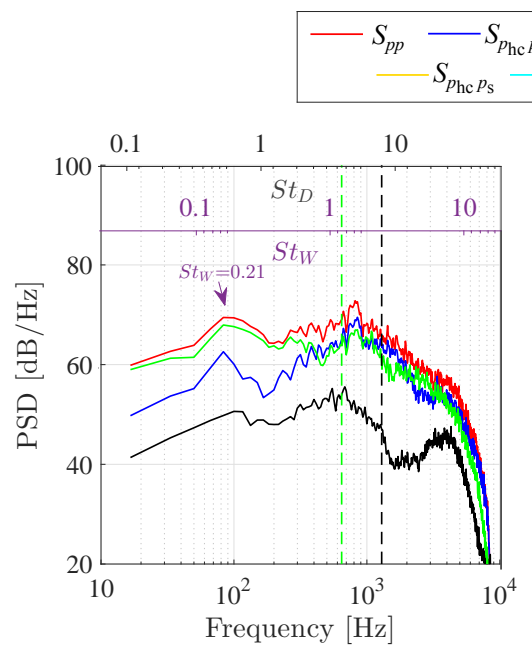

(a) PSD at the hub side.

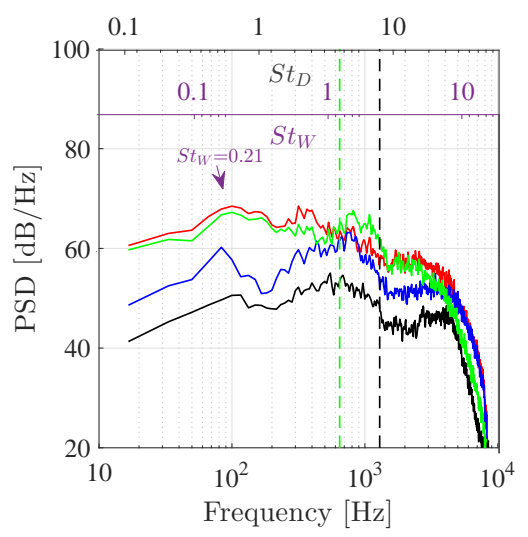

(c) PSD at the sidewall side.

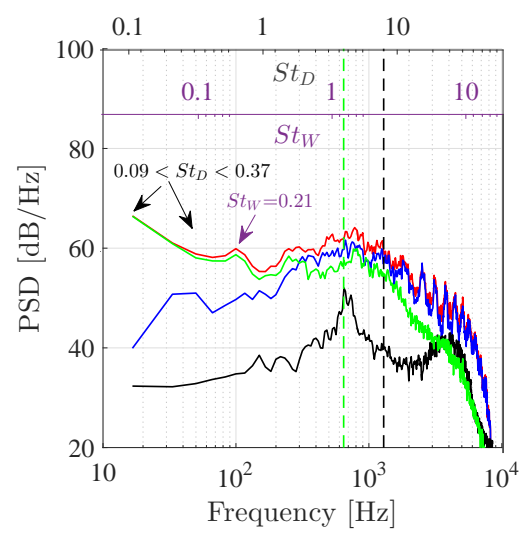

(e) PSD at the ground side.

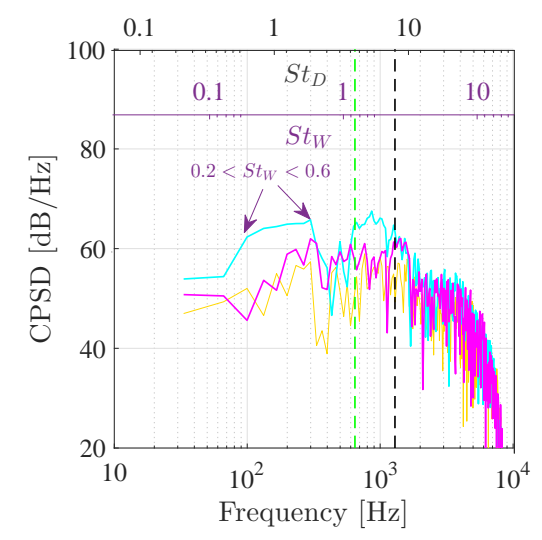

(b) CPSD at the hub side.

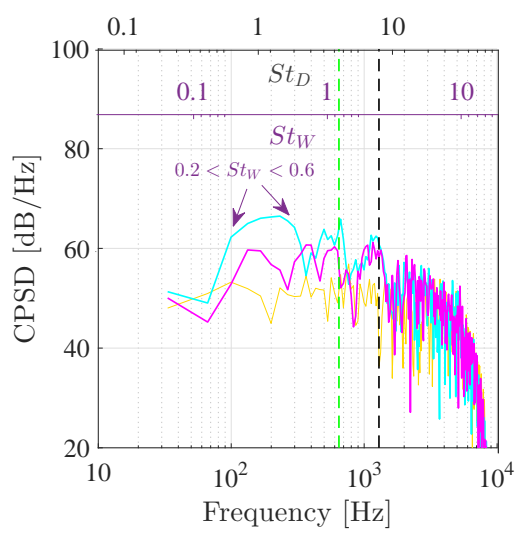

(d) CPSD at the sidewall side.

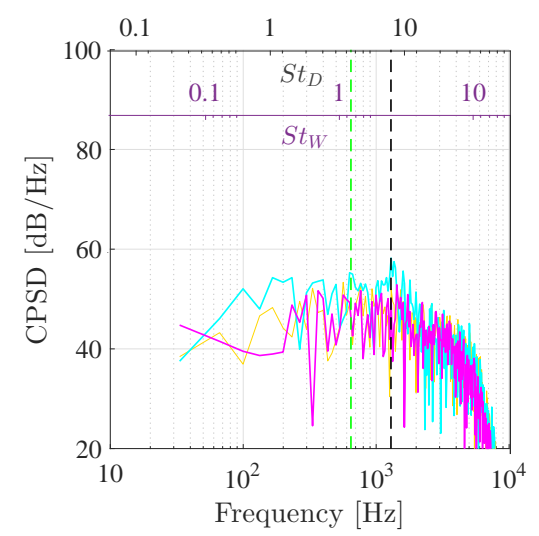

22 (f) CPSD at the ground side.

Figure 11: PSD and CPSD of far-field acoustic pressures from different parts of the wheel, against both frequency and two Strouhal numbers. - - - : first depth mode $(646 \mathrm{~Hz}) ;$ - - - : second depth mode $(1292 \mathrm{~Hz})$. $h c, s$ and $t$ are hub cavity, sidewall and tyre respectively. $p$ is the farfield acoustic pressure. 
the auto powers are discussed. The hub cavity auto power $S_{p_{\mathrm{hc}} p_{\mathrm{hc}}}$ at the frequencies around the two depth modes is higher at the hub side (approx. 70 $\mathrm{dB} / \mathrm{Hz}$ in Figure 11(a)) than the sidewall side (approx. $60 \mathrm{~dB} / \mathrm{Hz}$ in Figure 11(c)) and ground side (approx. $60 \mathrm{~dB} / \mathrm{Hz}$ in Figure 11(e)). This is because most of the hub cavity noise is radiated towards observers facing the hub. For the sidewall auto power $S_{p_{\mathrm{s}} p_{\mathrm{s}}}$, a spectral hump is present at $f=4000 \mathrm{~Hz}$, which is in the high frequency range and indicates that the small-scale flow features at the sidewall rim cavity, and their corresponding pressure fluctuations on the surface, are responsible for the far-field noise radiated at this frequency. The tyre auto power $\left(S_{p_{\mathrm{t}} p_{\mathrm{t}}}\right)$ shows a spectral hump at a Strouhal number based on the wheel width of $S t_{W}=0.21$. The tyre auto power at $S t_{W}=0.21$ is higher at the hub side (approx. $70 \mathrm{~dB} / \mathrm{Hz}$ ) and sidewall side (approx. $70 \mathrm{~dB} / \mathrm{Hz}$ ) than the ground side (approx. $60 \mathrm{~dB} / \mathrm{Hz}$ ), indicating 315 a directivity pattern corresponding to a force dipole aligned with the $z$ axis (side force). In Figure 11(e), the tyre auto power $S_{p_{\mathrm{t}} p_{\mathrm{t}}}$ is dominant at very low frequencies $(f<50 \mathrm{~Hz})$. This frequency range corresponds to a Strouhal number of $0.09<S t_{D}<0.37$. The tyre auto power is higher at the ground side $(>60 \mathrm{~dB} / \mathrm{Hz})$ than the hub side (approx. $60 \mathrm{~dB} / \mathrm{Hz}$ ) and sidewall side (approx. $60 \mathrm{~dB} / \mathrm{Hz}$ ). This indicates a directivity pattern corresponding to a force dipole aligned with the $y$ axis (lift force), which shows a lift dipole directivity pattern. The directivity patterns of the overall wheel are further discussed in Section 4.3.4. From the auto powers at the three observer angles, the tyre auto power $S_{p_{\mathrm{t}} p_{\mathrm{t}}}$ is dominant over the hub cavity auto power ${ }_{325} S_{p_{\mathrm{hc}} p_{\mathrm{hc}}}$ and the sidewall auto power $S_{p_{\mathrm{s}} p_{\mathrm{s}}}$ in the frequency range of $f<200$ $\mathrm{Hz}$. Therefore, the tyre is the main low frequency noise source. 
The cross powers (CPSD) are noisier than the auto powers due to the limited length of the FW-H signals. The real part of the cross power is a measure of the power shared at a given frequency by the farfield pressure angle $(\phi)$ in Figure 12. The definition of the angles is given in Figure 7. 11(b) and sidewall side observer in Figure 11(d), a spectral hump is shown by the Fourier transform of the cross-correlation between the hub cavity and tyre signals $\left(S_{p_{\mathrm{hc}} p_{\mathrm{t}}}\right)$ at $0.2<S t_{W}<0.6$, which is not present at the ground side observer in Figure 11(f). This spectral hump is the effect of the hub cavity on the tyre noise that dominates at low frequencies in this frequency range $\left(0.2<S t_{W}<0.6\right)$. A second spectral hump in the Fourier transform on the cross correlation between the hub cavity and tyre signals $\left(S_{p_{\mathrm{hc}} p_{\mathrm{t}}}\right)$ occurs between the first and second depth modes at the hub side observer in Figure 11(b). This does not occur at the sidewall observer in Figure 11(d) and ground side observer in Figure 11(f). This spectral hump around the depth modes is due to the interactions of hub cavity flow with the downstream tyre. For the ground side observer in Figure 11(f), the level of Fourier transform of the cross correction between the hub cavity and sidewall $\left(S_{p_{\mathrm{hc}} p_{\mathrm{s}}}\right)$, hub cavity and tyre $\left(S_{p_{\mathrm{hc}} p_{\mathrm{t}}}\right)$ and sidewall and tyre $\left(S_{p_{\mathrm{s}} p_{\mathrm{t}}}\right)$ are all lower compared to the hub side observer (Figure 11(b)) and the sidewall side observer (Figure11(d)). Since the ground side is the least efficient radiation direction for the hub cavity noise and sidewall noise, the coherent power of the acoustics from the three components is lower.

\subsubsection{Directivity}

The directivity of OASPL is plotted against polar angle $(\psi)$ and azimuthal 


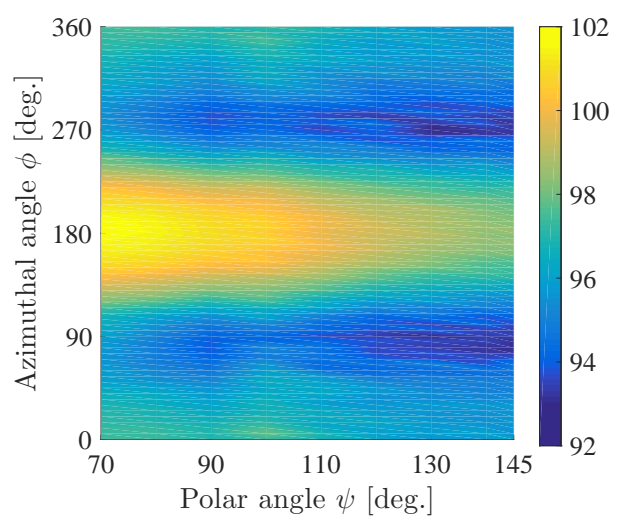

Figure 12: Directivity of OASPL $[\mathrm{dB}]$ against polar and azimuthal angles.

Most of the noise radiates towards the hub side $\left(\phi=180^{\circ}\right)$ in the upstream direction $\left(\psi<90^{0}\right)$.

The directivity of narrowband SPL (with a band width of $8 \mathrm{~Hz}$ ) at different frequencies is plotted in Figure 13 to give an insight into how the radiation patterns vary with frequency. Firstly at $20 \mathrm{~Hz}\left(S t_{D}=0.13\right)$ in Figure 13(a), the peak directivity is towards the wing side $\left(\phi=90^{\circ}\right)$ and the ground side $\left(\phi=270^{\circ}\right)$. As discussed in Section 4.3.3, the directivity at this frequency shows a lift dipole pattern (aligned with $y$ axis). At $90 \mathrm{~Hz}$ ${ }_{360}\left(S t_{W}=0.21\right)$ in Figure 13(b), the directivity still shows a dipole pattern but with the favourable directions changing to the sidewall side $\left(\phi=0^{0}\right)$ and hub side $\left(\phi=180^{\circ}\right)$, presenting a side force dipole pattern (aligned with the $z$ axis). As the frequency increases to $646 \mathrm{~Hz}$ (first depth mode) and 1292 $\mathrm{Hz}$ (second depth mode), the dipole behaviour disappears and there is only one single radiation direction towards the hub side. The noise generated at the first and second depth modes radiates more in the upstream direction towards the hub side, which determines the OASPL directivity in Figure 12. 


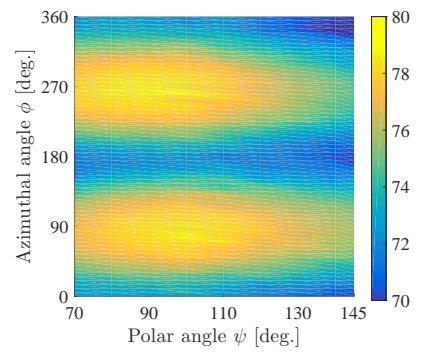

(a) $f=20 \mathrm{~Hz}$.

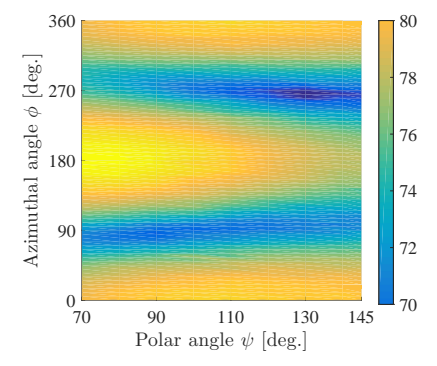

(b) $f=90 \mathrm{~Hz}$.

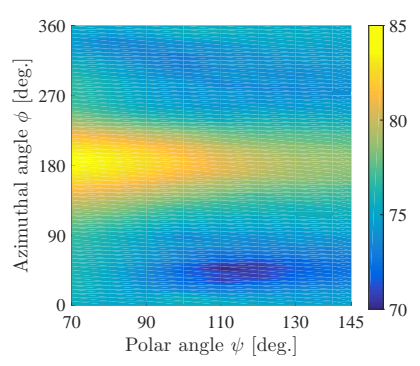

(c) $f=646 \mathrm{~Hz}$.

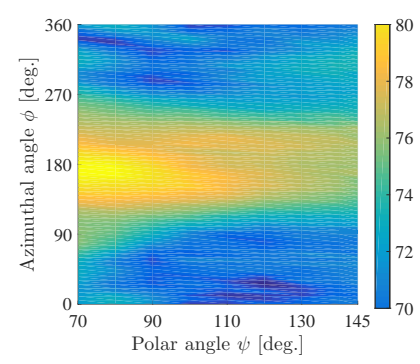

(d) $f=1292 \mathrm{~Hz}$.

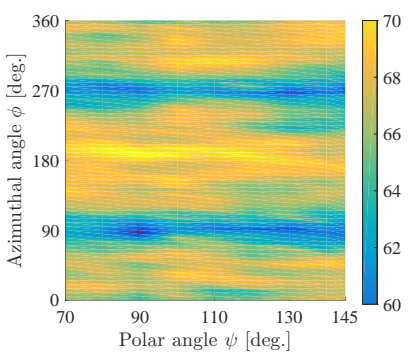

(e) $f=4000 \mathrm{~Hz}$.

Figure 13: Directivity of narrow band SPL [dB] against polar and azimuthal angles at different frequencies. 
At $4000 \mathrm{~Hz}$, the directivity is again dipole in nature, radiating towards both the sidewall and hub sides. As discussed in the previous section, this noise is

This will also be shown in Section 4.4, when the rim cavities are covered.

\subsubsection{Noise sources localization}

When the FW-H integral surface is coincident with the solid wheel surface and neglecting the quadrupole sources, the $\mathrm{FW}-\mathrm{H}$ equation in the frequency domain can be expressed as,

$$
p_{a}(\mathbf{x}, f)=\int_{\mathbf{S}} \mathcal{P}(\mathbf{y}, f) \mathrm{d} S
$$

where,

$$
\mathcal{P}(\mathbf{y}, f)=\frac{1}{4 \pi r} p_{w}(\mathbf{y}, f) \hat{\mathbf{n}} \cdot \hat{\mathbf{r}}
$$

where $\mathbf{x}$ and $\mathbf{y}$ are the observer and source locations and their distance is $r, S$ is the FW-H integral surface, $\hat{\mathbf{n}}$ is the unit outward normal vector to the surface $S$ and $\hat{\mathbf{r}}$ is the unit vector pointing from the source point $\mathbf{y}$ to the observer location $\mathbf{x}$. $\mathcal{P}(\mathbf{y}, f)$ is the noise source strength at each $\mathrm{FW}-\mathrm{H}$

panel on the integral surface, and thus can be used to indicate the noise source locations at different frequencies. The noise source strength converted to a $\log$ scale is therefore,

$$
\mathcal{N}(\mathbf{y}, f)=10 \log \left(\frac{\mathcal{P}^{2}(\mathbf{y}, f)}{p_{\text {ref }}^{2}}\right),
$$

where $p_{\text {ref }}$ is the reference pressure $(20 \mu \mathrm{Pa})$.

The plots of noise source strength $\mathcal{N}(\mathbf{y}, f)$ for far-field observers at the 385

hub side is provided in Figure 14, which shows that the dominant noise 
sources are the wall pressure fluctuations on the hub and downstream of the hub cavity. For low frequencies at $20 \mathrm{~Hz}$ and $90 \mathrm{~Hz}$, the rear part of the tyre is the major noise source. At the cavity depth modes at frequencies of $646 \mathrm{~Hz}$ and $1292 \mathrm{~Hz}$, there is a triangular region that originates from the

the leading edge of the hub rim cavity. This triangular region corresponds to the separation line in Figure 6 and the flow field in this region is highly turbulent. At a frequency of $4000 \mathrm{~Hz}$ in Figure 14(e), the dominant far-field noise is due to the pressure fluctuations around the hub and the downstream part of the hub rim cavity. An analysis of Coherent Output Power between wall pressures and far-field acoustic pressures has also been performed, which gives similar noise source patterns shown in Figure 14.

\subsection{Effects of covering the hub cavity and rim cavities}

The effect of covering the hub cavity and rim cavities on the farfield acoustic spectra is shown in Figure 15 for three different observer angles. The different configurations are shown in Figure 2. The NHC configuration is with the hub cavity covered. The NHCRC configuration is with the hub and rim cavities covered. At low frequencies, the three different configurations have similar levels at all three observer locations. Therefore the low frequency noise is mainly due to the tyre and the presence of the hub and rim cavities have relatively little effect on this low frequency noise. This is consistent with the discussion on the auto PSD in Figure 11. For the hub side observer in Figure 15(a), there is a significant reduction of noise in the middle frequency range around the hub cavity depth modes when the hub cavity is covered (NHC). Again this shows that the mid frequency noise around the first and second cavity depth modes is generated by the hub cavity. The high 


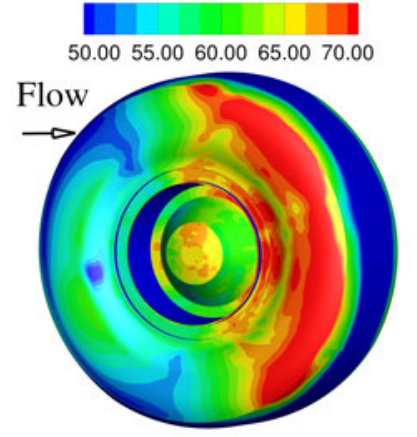

(a) $f=20 \mathrm{~Hz}$.

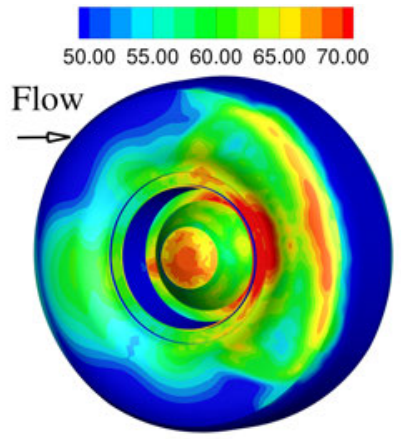

(b) $f=90 \mathrm{~Hz}$.

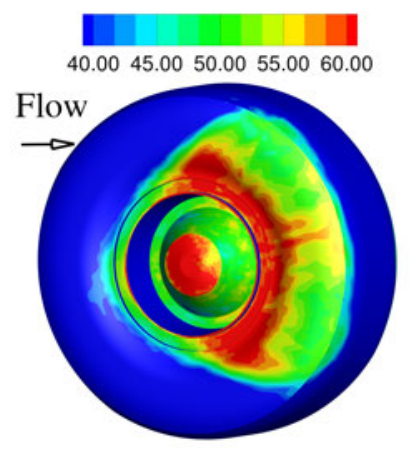

(c) $f=646 \mathrm{~Hz}$.

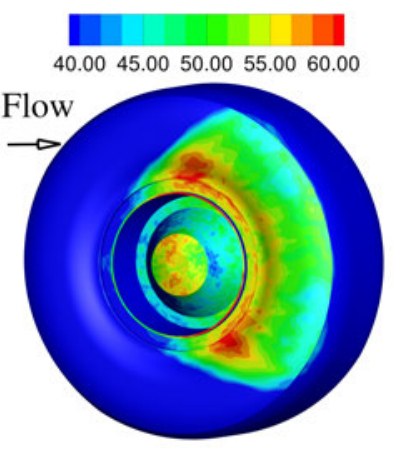

(d) $f=1292 \mathrm{~Hz}$.

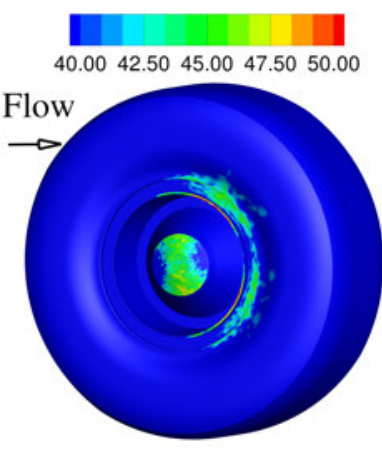

(e) $f=4000 \mathrm{~Hz}$.

Figure 14: The noise source strength $\mathcal{N}(\mathbf{y}, f)$ for the far-field observer at the hub side $\left(\psi=90^{\circ}, \phi=180^{\circ}\right)$. 
frequency noise is significantly decreased when the rim cavities are additionally covered (NHCRC). The high frequency noise is dominated by the flow generated by the rim cavities.

For the far-field observer at the sidewall side in Figure 15(b), there a

415 smaller differences between the baseline configuration and the configuration with the hub cavity covered (NHC) showing that the noise at the sidewall side is relatively less effected by the presence of the hub cavity. Covering both the hub cavity and the rim cavities (NHCRC configuration), results in a significant reduction in the high frequency noise. For the observer at the ground side in Figure 15(c), there is a reduction in the middle frequency range compared to the baseline and NHCRC cases. However, this reduction is not as significant as the hub side observer (Figure 15(a)). This is due to most of the hub cavity noise being radiating towards the hub side rather than the ground side. When the rim cavities are covered, a reduction of the high frequency noise at the ground side is also illustrated by the NHCRC configuration.

The OASPL for the three configurations are shown in Figure 16. Compared to the baseline configuration, when the hub cavity is covered (NHC configuration), a significant reduction of $6.4 \mathrm{~dB}$ is achieved at the hub side, most of which is from the middle frequency noise around the hub cavity depth modes, as shown in Figure 15(a). The far-field noise reductions achieved by covering the hub cavity at the sidewall side and ground side observers are less. The effect of the rim cavities on the far-field acoustics can be demonstrated by comparing the NHC case and the NHCRC case. This shows that most of the rim cavity noise is radiated towards the hub side and sidewall 


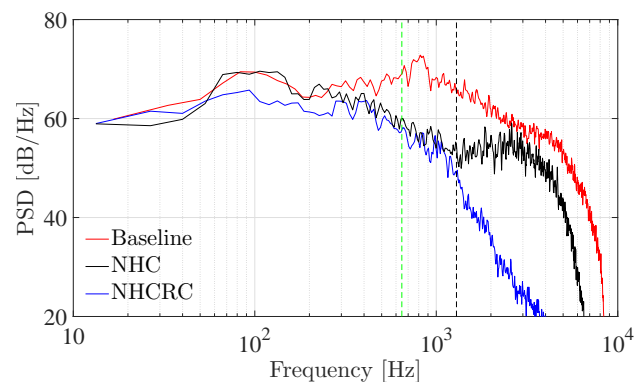

(a) Hub side $\left(\psi=90^{\circ}, \phi=180^{\circ}\right)$.

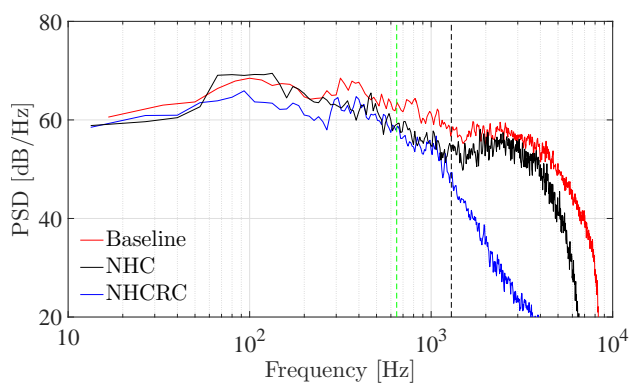

(b) Sidewall side $\left(\psi=90^{\circ}, \phi=0^{\circ}\right)$.

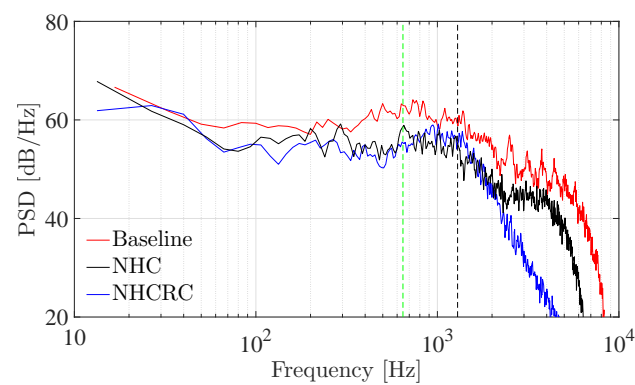

(c) Ground side $\left(\psi=90^{\circ}, \phi=270^{\circ}\right)$.

Figure 15: Comparisons of the far-field acoustic pressure spectra obtained from the simulations of the baseline configuration, No Hub Cavity (NHC) configuration and No Hub Cavity and Rim Cavities (NHCRC) configuration. - - - first depth mode (646 Hz), - - second depth mode $(1292 \mathrm{~Hz})$. 


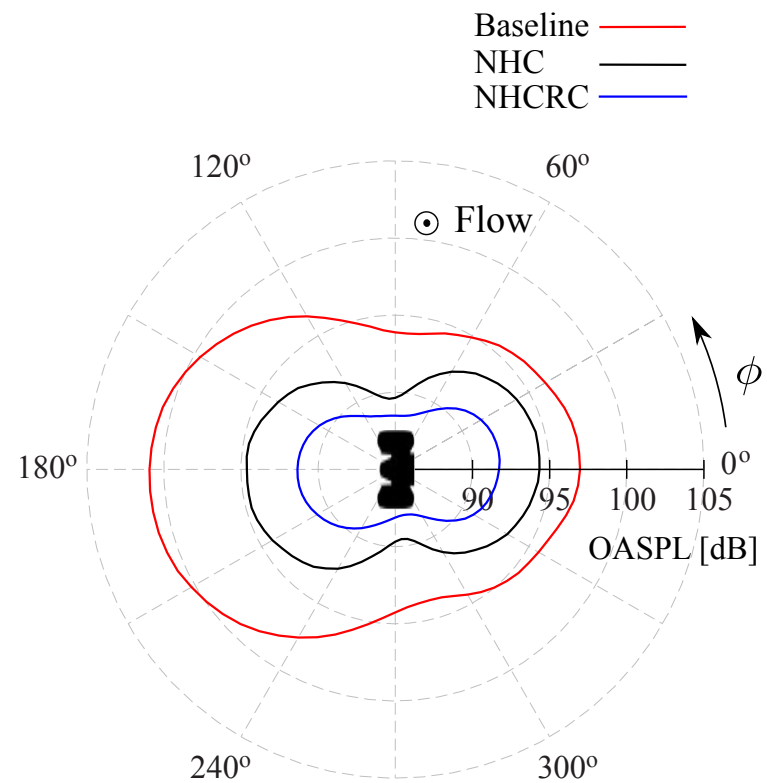

Figure 16: Comparisons of the far-field acoustic directivity obtained from different configurations.

side observers. As shown in Figure 15(a), 15(b) and 15(c), most of the reductions by additionally covering the rim cavities (NHCRC configuration) are in the high frequency range. Compared with the baseline configuration, the NHCRC configuration shows reductions of $10.3 \mathrm{~dB}, 6.1 \mathrm{~dB}$ and $5.8 \mathrm{~dB}$ at the hub side, sidewall side and ground side observers respectively.

\section{Conclusions}

High-order CAA simulations were performed to investigate the flow features and far-field acoustics of three different landing gear wheel configurations to isolate the effects of a hub cavity and rim cavities on landing gear wheel noise. The baseline configuration is the same with the geometry used in the experimental work by Zhang et al. [14]. The numerical methodol- 
ogy applied to the baseline case has been validated previously by Wang et al. [15]. The surface flow topology on the side face shows that there are separations and attachments in the baseline and the configuration with the hub cavity covered, whereas the flow is fully attached to the side face for the configuration when both the hub cavity and rim cavities are covered.

The analysis of far-field acoustic pressure spectra of the baseline configuration shows that a significant noise source is present in the middle frequency range around $646 \mathrm{~Hz}$ and $1292 \mathrm{~Hz}$, related to the first and the second depth modes of the hub cavity geometry. This middle frequency noise is fixed by the cylindrical cavity dimensions and does not scale with flow velocities. Higher coherence between the hub side far-field acoustics and the wall pressure fluctuations around the hub cavity region is demonstrated at the two depth mode frequencies. The acoustic predictions using different parts of the wheel surface show that the tyre is the main low frequency noise source, and a lift and a side force dipole is present at $0.09<S t_{D}<0.37$ and $S t_{W}=0.21$, which is also demonstrated by the analysis of far-field acoustic directivity at different frequencies. The directivity of OASPL shows that more noise is radiated towards the hub side observer. Most of the contribution to the OASPL comes from the hub cavity noise in the middle frequency range. The noise source strength function, which is obtained from the FW-H equation in the frequency domain, shows the hub cavity area is the dominant noise source in this frequency range.

The configurations with the hub cavity covered (NHC) and with both 470 the hub cavity and rim cavities covered (NHCRC) show that the tyre, hub cavity and rim cavities are the main noise sources in the low, middle and 
high frequency ranges, respectively. When the hub cavity and rim cavities are covered, reductions of $10.3 \mathrm{~dB}, 6.1 \mathrm{~dB}$ and $5.8 \mathrm{~dB}$ in the far-field acoustic OASPL are achieved at the hub side, sidewall side and ground side observers respectively.

\section{Acknowledgement}

This work has been financially supported by an Airbus/UK Engineering and Physical Sciences Research Council (EPSRC) industrial CASE studentship. All data supporting this study are openly available from the 480 University of Southampton repository at http://doi.org/10.5258/SOTON/ 403575. 


\section{References}

[1] Y. Guo, K. J. Yamamoto, and R. W. Stoker, "An empirical model for landing gear noise prediction," in 10th AIAA/CEAS Aeroacoustics Conference, AIAA 2004-2888, 2004.

[2] W. Liu, J. W. Kim, X. Zhang, D. Angland, and B. Caruelle, "Landinggear noise prediction using high-order finite difference schemes," Journal of Sound and Vibration, vol. 332, no. 14, pp. 3517-3534, 2013.

[3] D. P. Lockard, M. Khorrami, and F. Li, "Aeroacoustic Analysis of a Simplified Landing Gear," in 10th AIAA/CEAS Aeroacoustics Conference, AIAA 2004-2887, 2004.

[4] M. Zdravkovich, V. Brand, G. Mathew, and A. Weston, "Flow past short circular cylinders with two free ends," Journal of Fluid Mechanics, vol. 203, pp. 557-575, 1989.

495

[5] B. S. Lazos, "Mean Flow Features Around the Inline Wheels of FourWheel Landing Gear," AIAA Journal, vol. 40, no. 2, pp. 193-198, 2002.

[6] B. Lazos, "Surface Topology on the Wheels of a Generic Four-Wheel Landing Gear," AIAA Journal, vol. 40, no. 12, pp. 2402-2411, 2002.

[7] D. H. Neuhart, M. R. Khorrami, and M. M. Choudhari, "Aerodynamics of a Gulfstream G550 nose landing gear model," in 15th AIAA/CEAS Aeroacoustics Conference, AIAA 2009-3152, 2009.

[8] Y. Yokokawa, T. Imamura, H. Ura, H. Uchida, H. Kobayashi, and K. Yamamoto, "Experimental study on noise generation of a two-wheel main 
landing gear," in 16th AIAA/CAES Aeroacoustics Conference, AIAA 2010-3973, 2010.

[9] P. Spalart and K. Mejia, "Analysis of Experimental and Numerical Studies of the Rudimentary Landing Gear," in 49th AIAA Aerospace Sciences Meeting including the New Horizons Forum and Aerospace Exposition, AIAA 2011-0355, 2011.

[10] P. R. Spalart and D. A. Wetzel, "Rudimentary landing gear results at the 2012 BANC-II airframe noise workshop," vol. 14, no. 1, pp. 193-216, 2015 .

[11] S. M. Jaeger, N. J. Burnside, P. T. Soderman, W. C. Horne, and K. D. James, "Microphone Array Assessment of An Isolated 26\%-Scale, HighFidelity Landing Gear," in 8th AIAA/CEAS Aeroacoustics Conference, AIAA 2002-2410, 2002.

[12] D. Casalino, A. Ribeiro, E. Fares, and S. Noelting, "Lattice Boltzmann Aeroacoustic Analysis of the LAGOON Landing-Gear Configuration," AIAA Journal, vol. 52, no. 6, pp. 1232-1248, 2014.

[13] D. Casalino, A. F. Ribeiro, and E. Fares, "Facing rim cavities fluctuation modes," Journal of Sound and Vibration, vol. 333, no. 13, pp. 2812-2830, 2014 .

[14] X. Zhang, Z. K. Ma, M. Smith, and M. Sanderson, "Aerodynamic and Acoustic Measurements of a Single Landing Gear Wheel," in 19th AIAA/CEAS Aeroacoustics Conference, AIAA 2013-2160, 2013. 
[15] M. Wang, D. Angland, X. Zhang, and R. Fattah, "High-Order Numerical Simulations of An Isolated Landing Gear Wheel with A Hub Cavity," in 22nd AIAA/CEAS Aeroacoustics Conference, AIAA 2016-2902, 2016.

[16] O. Marsden, C. Bailly, C. Bogey, and E. Jondeau, "Investigation of flow features and acoustic radiation of a round cavity," Journal of Sound and Vibration, vol. 331, no. 15, pp. 3521-3543, 2012.

[17] J. Rossiter, "Wind-tunnel Experiments on the Flow Over Rectangular Cavities at Subsonic and Transonic Speeds," Technical Report 3438, Aeronautical Research Council Reports and Memoranda, 1964.

[18] O. Marsden, C. Bogey, and C. Bailly, "Depth effects on the flow features and noise signature of shallow cylindrical cavities at a Mach number of 0.25," in 18th AIAA/CEAS Aeroacoustics Conference, AIAA 2012-2232, 2012 .

[19] P. R. Spalart, S. Deck, M. L. Shur, K. D. Squires, M. K. Strelets, and A. Travin, "A New Version of Detached-eddy Simulation, Resistant to Ambiguous Grid Densities," Theoretical and Computational Fluid Dynamics, vol. 20, no. 3, pp. 181-195, 2006.

[20] J. W. Kim, "Optimised boundary compact finite difference schemes for computational aeroacoustics," Journal of Computational Physics, vol. 225, no. 1, pp. 995-1019, 2007.

[21] J. W. Kim, "High-order compact filters with variable cut-off wavenumber and stable boundary treatment," Journal of Computers and Fluids, vol. 39, no. 7, pp. 1168-1182, 2010. 
[22] M. Wang, R. Fattah, D. Angland, and X. Zhang, "High-Order Hy-

[28] N. Curle, "The influence of solid boundaries upon aerodynamic sound," Proceedings of the Royal Society of London A: Mathematical, Physical and Engineering Sciences, vol. 231, no. 1187, pp. 505-514, 1955. 